\title{
Classificação automática do tipo de ferro fundido utilizando reconhecimento de padrões em imagens de microscopia
}

\author{
Automatic classification of type of cast \\ iron using pattern recognition in \\ microscopy images
}

Douglas de Araújo Rodrigues ${ }^{1}$, Gesilane Pereira dos Santos ${ }^{1}$, Marcelo Correia Fernandes ${ }^{1}$ José Ciro dos Santos ${ }^{1}$, Francisco Nélio Costa Freitas ${ }^{1}$, Pedro Pedrosa Rebouças Filho ${ }^{1}$

\footnotetext{
${ }^{1}$ Laboratório de Processamento de Imagens e Simulação Computacional (LAPISCO), Instituto Federal de Educação, Ciência e Tecnologia do Ceará (IFCE) - CEP: 61925-315, Maracanaú - CE. e-mail: douglas@lapisco.ifce.edu.br, gesilane@lapisco.ifce.edu.br, marcelofernandes@lapisco.ifce.edu.br, pedrosarf@ifce.edu.br,ciro@ifce.edu.br, fneliocf@ifce.edu.br.
}

\section{RESUMO}

Os ferros fundidos possuem uma microestrutura, na qual, o formato da grafita presente tem direta influência na classificação entre os diversos tipos deste material. A classificação é feita, tradicionalmente, a partir da análise visual realizada por um especialista através do auxílio de um microscópio ótico. O presente trabalho propõe a utilização da Inteligência Computacional Aplicada em conjunto com extratores de informações em imagens de metalografia. A finalidade é auxiliar profissionais da área das Ciências dos Materiais na classificação de ferros fundidos de maneira automática e reduzir o tempo de classificação, restringindo o máximo possível as falhas presentes durante a classificação. Foram realizadas duas etapas para a análise. Na primeira foram consideradas as grafitas separadamente. Em uma segunda etapa, a imagem completa foi analisada considerando todas as grafitas extraídas da mesma, em que o tipo de objeto com maior incidência na análise seria o reconhecimento adotado para toda a amostra. Em ambas as etapas o classificador Support Vector Machine obteve os melhores resultados no reconhecimento do tipo de ferro fundido, com resultados próximos a 100\%, e com redução média do tempo de classificação em 92\%. Tanto os resultados como o tempo das classificações são comparados com a análise do especialista, como também aos resultados obtidos em classificações do ferro fundido que utilizam uma abordagem com redes neurais e uma classificação supervisionada utilizando apenas os descritores de forma. A partir dos resultados apresentados, conclui-se que a abordagem é promissora podendo incorporar softwares comerciais para auxiliar especialistas da área.

Palavras-chave: Ferro fundido, Processamento Digital de Imagens, Reconhecimento de Padrões, Inteligência Computacional Aplicada.

\begin{abstract}
The cast iron has a microstructure in which the shape of the contained graphite has direct influence in the classification between the several types of this material. The classification is usually made through a visual analysis performed by a specialist using an optical microscope. This work proposes the use of Applied Computational Intelligence in conjunction with extractors of information in metallographic images. The purpose is to assist professionals in the field of Materials Science in the classification of cast iron automatically and reduce the time for classification, restricting as much as possible the faults presented during classification. Two steps were performed for the analysis. In the first one we considered the graphites separately. In a second step, we analyzed the complete image considering all the graphite extracted from it, in which the type of object with greater incidence in the analysis would be the recognition adopted for the whole sample. In both steps, the classifier Support Vector Machine obtained the best results in the recognition of the type of cast iron, with results close to $100 \%$, with a mean reduction of the classification time by $92 \%$. Both the results and the time of the classifications are compared with the specialist's analysis, as well as the results obtained in cast iron classifications that use a neural network approach and a supervised classification using only the
\end{abstract}


shape descriptors. From the results presented, we concluded that the approach is promising and can incorporate commercial software to assist specialists in the field.

Keywords: Cast Iron, Digital Image Processing, Pattern Recognition, Applied Computational Intelligence.

\section{INTRODUÇÃO}

Os ferros fundidos são utilizados em larga escala pela indústria de máquinas e equipamentos, indústria automobilística, ferroviária, naval e outras. Esses materiais estão presentes em peças automotivas [1], em equipamentos de aquecimento de água [2] e em carcaças de rolamentos de esferas de discos de moagem [3]. A sua diversidade de aplicações se deve, principalmente, ao fato das propriedades mecânicas deste material variarem de acordo com a sua composição e ao tratamento térmico a qual é submetido.

CHIAVERINI [4] define o ferro fundido como uma liga ferro-carbono-silício, de teores de carbono geralmente acima de 2,0\%, em quantidade superior à que ser retida em solução sólida na austenita, de modo a resultar em carbono parcialmente livre.

O carbono presente na liga, geralmente, se apresenta na forma de grafita, que, por sua vez, possui formas variadas. Com base nessa diferenciação, CALLISTER [5] classifica o ferro fundido em diferentes tipos, entre eles se destacam os ferros fundidos cinzento, nodular e maleável. As fotomicrografias para esses tipos de ferros fundidos são apresentadas na Figura 1.

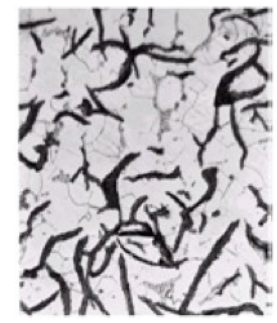

a)

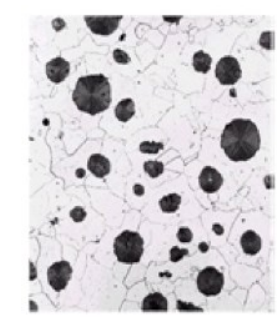

b)

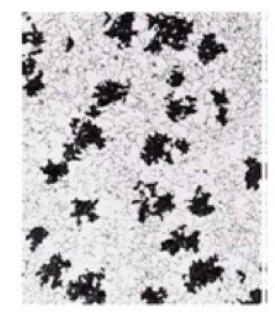

c)

Figura 1: Amostras de Ferros Fundidos: a) cinzento; b) nodular; c) maleável; [5]

O ferro fundido cinzento, no qual, o carbono presente encontra-se no formato de partículas laminares ou em veios, pode ser utilizado em fundições de motores a diesel, revestimentos e caixas de transmissão [5]. O ferro fundido nodular, também conhecido como dúctil, confere ao material características com alto módulo de elasticidade e resistência mecânica, e apresenta carbono livre na forma de grafita esferoidal. As aplicações típicas deste tipo de material são em corpos de válvula de bombas, engrenagens e cilindros[6]. Já as aplicações do ferro fundido maleável estão envolvidas nos serviços gerais de engenharia sob temperaturas normais e elevadas. As suas grafitas são apresentadas na forma de flocos ou vermicular [5].

As características inerentes a cada tipo desse material exigem uma classificação minuciosa e coerente com a realidade, para que não haja nenhum equívoco em seu uso, seja ele industrial ou laboratorial. Porém, essa classificação nem sempre condiz com a realidade, visto que é necessário um longo período de experiência para que profissionais possam realizar esse trabalho de maneira pertinente e com o mínimo de falhas.

A automatização do processo convencional de análise das grafitas, que compõem os ferros fundidos, visa uma considerável diminuição de erros provenientes da manipulação do homem na amostra, além de otimizar o tempo gasto na realização de sua classificação.

Diversas técnicas de Processamento Digital de Imagens (PDI) e de reconhecimento de padrões têm sido empregadas em vários ramos das Ciências dos Materiais afim de auxiliar profissionais e especialistas em suas atividades diárias. Em estudos de microscopia ótica são comumente usados na caracterização microestrutural $[7,8,9,10]$ e na análise dos ferros fundidos $[11,12,13,14]$, como nos trabalhos realizados para o cálculo da densidade de nódulos de grafita $[15,16]$ e na quantificação de microestruturas em metais utilizando redes neurais artificiais [17].

Neste contexto, surgiram trabalhos que visam classificar de forma automática os ferros fundidos. Dentre estes, vale destacar os trabalhos de GOMES e PACIONIRK [18] e PATTAN et al. [19]. PATTAN et al. [19] realizaram a classificação do ferro fundido, com base na morfologia dos grãos de grafita usando uma abordagem com redes neurais. Para o treinamento e o teste das redes, foram usadas as formas de grão identificadas em desenhos presentes na norma ISO-945 de 2008 e a classificação de grãos pelos especialistas. 
Em seu trabalho GOMES e PACIORNIK [18] desenvolveram um sistema computacional que tem por finalidade classificar amostras de ferro fundido com base na análise de seus veios, lamelas ou nódulos. No qual, cada uma das figuras apresentadas pela norma ISSO-945 passou pelo processo de extração de atributos, suas características mais representativas geraram os modelos utilizados para a construção do conjunto de treinamento, um classificador então é o responsável por categorizar novas imagens de ferros fundidos em uma das classes, com base no vetor de características dessas novas imagens e nas informações aprendidas durante o processo de treinamento do software.

Neste sentindo, o presente trabalho propõe a utilização de Inteligência Computacional Aplicada e Reconhecimento de Padrões na análise digital de imagens metalográficas, destinadas a classificação e caracterização de ferros fundidos. O objetivo é automatizar o processo de análise, reduzindo de forma apreciável, o tempo necessário e a possibilidade de falhas no resultado da interpretação.

\section{MATERIAIS E MÉTODOS}

Nesta seção são apresentados os métodos de Processamento Digital de Imagens e Reconhecimento de Padrões utilizados na abordagem de classificação de ferro fundido proposto neste trabalho, bem como as métricas usadas na avaliação dos resultados.

\subsection{PROCESSAMENTO DIGITAL DE IMAGENS}

Nesta subseção, serão explicadas as etapas de segmentação dos objetos em cada imagem e os atributos extraídos destes objetos para posterior classificação.

\subsubsection{Segmentação}

Um sistema de Visão Computacional (VC) é geralmente constituído pelas etapas de aquisição das imagens, pré-processamento, segmentação, extração de atributos e reconhecimento de objetos. A segmentação é a etapa responsável por dividir a imagem em regiões de interesse, tendo fundamental importância em um sistema de $\mathrm{VC}$, em que o desempenho deste sistema depende essencialmente da qualidade desta etapa [20].

De modo geral, existem duas categorias de segmentação que se diferenciam, sobretudo, pela propriedade em que se baseiam. São estes os de segmentação por descontinuidade e por similaridade. A primeira categoria realiza a divisão da imagem com base na detecção de mudanças bruscas entre pixels vizinhos na imagem, e são responsáveis pela deteç̧ão de pontos, linhas e bordas. A segmentação por similaridade baseiase na separação de objetos por possuírem valores próximos de uma dada característica preestabelecida [21].

Para se obter um desempenho desejável nesta etapa, alguns métodos, ou combinações entre eles, são bastante aplicados. Neste trabalho, foi utilizada a segmentação por Contornos Conectados, que utiliza conceitos de segmentação por descontinuidade.

Na segmentação por meio dos Contornos Conectados, inicialmente é necessária a aplicação do gradiente detector de bordas Canny na imagem trabalhada em níveis de cinza. Este detector apresenta as posições das descontinuidades de intensidade controlada que representam as bordas detectadas [22]. Essas posições são alocadas em um vetor bidimensional [23]. A partir daí, é realizada uma análise de busca desses vetores. Esta verificação tem por objetivo encontrar, no sentido horário, o próximo pixel vizinho conectado. Este processo se repete até que não existam mais pixels vizinhos a este ou até o último pixel analisado esteja conectado ao pixel inicial, como notado na Figura 2. Após este processo é efetuado uma aproximação poligonal, que consiste na alocação em um novo vetor de elementos presentes na invariação do contorno.

\subsubsection{Extração de características}

Essa etapa visa extrair as características da região de interesse das imagens resultantes do processo de segmentação. Estas imagens são representadas numericamente de modo a facilitar a aplicação do processo seguinte, que corresponde a classificação por meio da aprendizagem de máquinas. Os extratores usados neste trabalho são descritos abaixo.

Os Momentos Centrais são provenientes da redução dos Momentos Espaciais com o centro de gravidade do objeto. Desde modo, os Momentos Centrais referem-se ao centro de gravidade do objeto. Este extrator apresenta a vantagem de invariância à translação, o que torna possível a descrição da forma do objeto [24].

Nos Momentos Estatísticos a extração de atributos é realizada a partir da distribuição na imagem em níveis de cinza que, são usualmente calculadas com base no histograma. Desde modo, essas características possibilitam a descrição estatística da relação entre os níveis de cinza [21, 25]. 
O reconhecimento independentemente da posição, tamanho e orientação é o princípio dos Momentos de Hu. HU [25] apresentou em sua teoria, uma possibilidade capaz de realizar a descrição de figuras geométricas planas com base nos momentos invariantes bidimensionais [26, 27, 28].

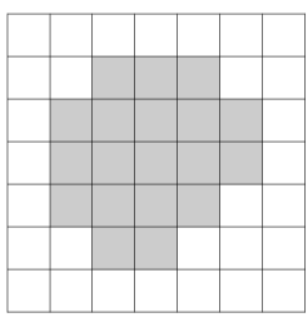

(a)

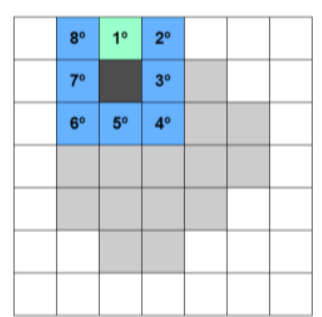

(b)

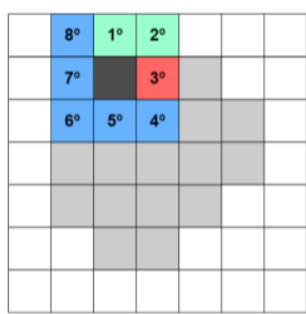

(c)

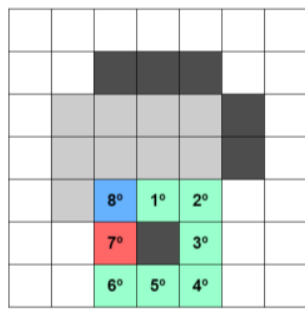

(d)

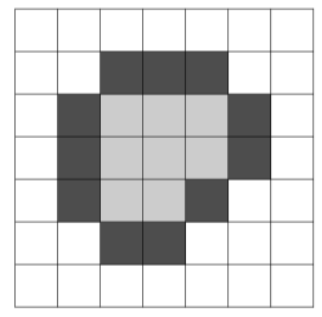

(e)

Figura 2: Ilustração da aplicação da segmentação por Contornos Conectados: a) imagem de entrada; b) imagem com primeiro ponto encontrado em preto; c) imagem com o segundo ponto encontrado em vermelho; d) processo; e) resultado final.

HARALICK [29] apresentou uma forma para descrever as texturas baseando-se em estatísticas de segunda ordem. Os recursos decorrentes de cálculos de matrizes de co-ocorrência (GLCM - Gray Level Cooccurrence Matrix) são definidos no lugar de uma imagem em determinado sentido. Estas matrizes consistem em uma contagem da quantificação de combinações diferentes de níveis de cinza e são fundamentais, pois elas são a base para a preparação de várias medidas estatísticas conhecidas como Descritores Haralick [30, 31, 32].

Outro extrator que objetiva realizar a descrição de texturas é o operador LBP (Library Binary Pattern).

Este tem como fundamentação atribuir um rótulo para cada pixel da imagem a partir de uma limiarização adaptativa local, obtendo como resultado um número binário para cada pixel. Sendo assim, o histograma está pronto para a descrição da textura. A princípio, utilizava-se o kernel 3x3 para a realização da limiarização. Com a necessidade da descrição de texturas de escalas diversas fez necessário que o operador LBP trabalhasse com diferentes kernels [33].

\section{RECONHECIMENTO DE PADRÕES}

Nesta subsecção, há a definição das cinco técnicas de reconhecimento de padrões usadas neste trabalho.

\subsubsection{Reconhecimento de Padrões usando os K-Nearest Neighbors}

O algoritmo do classificador K-Nearest Neighbors (KNN) analisa os padrões que estão vizinhos um dos outros no espaço das características e conclui que estes pertencem ao mesmo conjunto padrão. Deste modo, os objetos que possuem características semelhantes pertencem ao mesmo grupo. Essa análise pode considerar uma faixa de um até k vizinhos. Sendo que cada vizinho indica uma classe. Quando a observação com um número de vizinhos é superior a 1, há para cada ponto de teste a previsão de diferentes valores. Estas previsões são efetuadas de forma diferente em problemas de classificação e de regressão [34]. Desde modo, tem por finalidade a classificação do ponto de teste.

Em problemas de classificação, em que a classe assume valores em conjuntos discretos, a maioria das classes que foram votadas por cada vizinho é adotado para classificar o ponto de teste. Quando se apresenta problemas de regressão, na qual os conjuntos se apresentam de forma continua, as previsões do KNN são baseadas em uma média ou na mediana dos resultados nos $\mathrm{k}$ vizinhos mais próximos. 


\subsubsection{Reconhecimento de padrões usando o K-Means}

Frequentemente, o K-Means é utilizado para a descoberta de agrupamentos de dados, porém, pode ser adaptado para aplicações em classificação de padrões. O algoritmo K-Means particiona um conjunto de dados em $\mathrm{k}$ agrupamentos, de maneira que cada um possua um centróide [35]. Este conjunto de centróides são os vetores que representam o conjunto de dados. Assim um conjunto de dados pode ser compactado para este conjunto de $\mathrm{k}$ vetores.

Inicialmente é realizado um treinamento não-supervisionado, da qual, posteriormente, os padrões são associados aos centróides mais próximos, de tal maneira que ao final deste processo estão associados $\mathrm{k}$ padrões a um determinado centróide. Em seguida, cada centróide recebe o rótulo da classe com mais ocorrências dentre os seus k padrões associados. Quando um exemplo não visto é apresentado, o K-Means verifica qual o centróide mais próximo e então atribui a esta amostra o rótulo deste centróide.

\subsubsection{Reconhecimento de Padrões usando o Bayes}

O classificador Bayes utiliza uma abordagem em fundamentos estatísticos e categoriza um objeto com base na probabilidade de este pertencer a uma dada classe. O classificador calcula as probabilidades de um objeto pertencer a uma das classes possíveis, determinando para este, a classe que obteve a maior dessas probabilidades, isto é, a classe mais provável.

O teorema de decisão Bayesiano faz uso da probabilidade de vários objetos que pertencerem a mesma

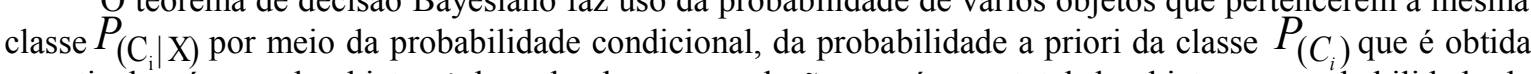
a partir do número de objetos $i$ de cada classe em relação ao número total de objeto, e a probabilidade de ocorrência desses objetos $P_{(X)}$ [36]. Esta teoria é descrita na Fórmula de Bayes na Equação 1.A partir desta fórmula, modifica-se a probabilidade a priori tendo em vistas novas evidências de forma a obter probabilidades a posteriori.

$$
P_{\left(\mathrm{C}_{\mathrm{i}} \mid \mathrm{X}\right)}=\frac{P_{\left(X \mid C_{i}\right)} P_{\left(C_{i}\right)}}{P_{(X)}}
$$

\subsubsection{Reconhecimento de Padrões usando Support Vector Machine}

Com base na Teoria de Aprendizado Estatístico [37] que também é conhecida como a Teoria de VapnikChervonenkis (VC), as Support Vector Machine (SVMs) são utilizadas para a classificação e análise de regressão. Embasadas no princípio da minimização do risco estrutural, o processo de otimização do SVM visa determinar uma função que separa durante a realização do trade-off o que existe entre generalização e sobreajuste.

Um aspecto comum do SVM consiste em uma formulação que destina a resolução de problemas binários. Apesar de o SVM ter sido formulado para problemas com duas classes, o mesmo possui abordagens para resolver problemas de multiclasses [38]. As abordagens de um-contra-um e um-contra-todos [39] são aqueles amplamente utilizadas.

Em problemas que apresentam exemplos não linearmente separáveis, o artificio do kernel é usado para gerar versões não-lineares do classificador SVM linear padrão. O kernel representa um produto de ponto no espaço de recurso em que os vetores originais são mapeados.

\subsubsection{Reconhecimento de Padrões usando o Multi-layer Perceptron (MLP)}

O Multilayer Perceptron (MLP) consiste em camadas de Rede Neural de Multicamadas que visam resolver problemas cujos padrões não são linearmente separáveis. As estruturas que compõem essa Rede são normalmente subdivididas em camada de entrada de rede, uma ou mais camadas ocultas e uma camada de saída [40, 41].

Esse classificador busca resolver problemas cujos padrões não são linearmente separáveis [42]. Estas redes estão divididas em duas etapas: treinamento e teste. A primeira etapa consiste no treinamento, na qual os pesos sinápticos são ajustados através de um algoritmo de treinamento da rede. Este algoritmo tem por finalidade treinar a rede de forma que se possa separar as classes apresentadas e, consequentemente, obter saídas desejadas [43]. A etapa de teste consiste no reconhecimento de padrões apresentados à rede que não estavam no conjunto de treinamento. 


\section{MATRIZ DE CONFUSÃO E MÉTRICAS DE AVALIAÇÃO}

Uma das ferramentas mais eficaz para a avaliação de um classificador é a matriz de confusão. Essa matriz possibilita realizar uma comparação entre os dados que são utilizados no teste e os que são utilizados para o treinamento, indicando o percentual de acerto e erro de classificação.

Na matriz de confusão, os dados são organizados em uma tabulação cruzada entre a classe prevista pelo classificador e a classe real das amostras. Os valores constantes na diagonal principal indicam os acertos enquanto que os demais dados são referentes aos erros de classificação. Desde modo, um bom classificador é aquele que apresenta altos valores na diagonal principal e baixos valores nos demais elementos da matriz de confusão.

Com base nas informações da matriz de confusão são calculadas as métricas utilizadas para avaliação dos classificadores: acurácia (Acc), sensibilidade (Sens), PPV (Positive Predictive Values) e o F-score. Estas métricas são dadas por:

$$
\begin{aligned}
& A c c=\frac{V P+V N}{V P+V N+F P+F N} \\
& \text { Sens }=\frac{V P}{V P+F N} \\
& P P V=\frac{V P}{V P+F P} \\
& F-\text { score }=\frac{(2 \cdot \text { Sens } \cdot P P V)}{(\text { Sens } \cdot P P V)}
\end{aligned}
$$

Onde, VP, FN, VN E FP são, respectivamente, os valores dos verdadeiros positivos, falsos negativos, verdadeiros negativos e falsos positivos.

Os valores dos verdadeiros positivos e falsos negativos representam, respectivamente, o número de grafitas de ferro fundido de uma determinada classe corretamente e incorretamente classificadas. Os verdadeiros negativos são o número de grafitas não pertencentes a uma determinada classe classificadas como não pertencentes a esta classe. Os valores dos falsos positivos são os números de grafitas incorretamente classificadas como pertencendo a uma determinada classe.

A acurácia revela a proporção de predições realizadas de forma correta. Essa métrica é calculada por meio da razão entre o número de grafitas classificadas corretamente e o número total de grafitas analisadas. A acurácia é uma das medidas mais importantes para decidir a escolha do classificador.

A sensibilidade é a taxa com que as amostras de ferros fundidos pertencentes a uma classe foram corretamente classificadas se comparadas ao total de amostras dessa classe. Essa métrica é calculada pela divisão dos acertos na classificação das grafitas de uma só classe pelo número total de amostras dessa classe. A métrica PPV, também conhecida como precisão, indica a taxa com que cada amostra de grafita de ferro fundido é classificada como pertencente a uma classe que realmente pertence àquela classe. Essa métrica é calculada através da divisão do número total de acertos na classificação da grafita pelo número total de predições dessa classificação. O F-score pode ser interpretado como uma média harmônica da sensibilidade e do PPV.

\section{METODOLOGIA}

Nesta seção é descrita a abordagem proposta neste trabalho para identificação e reconhecimento de grafitas de forma automática em imagens metalográficas. O processo adotado desde a aquisição até o reconhecimento de ferro fundido em uma amostra é apresentado no fluxograma da Figura 3. O primeiro passo é realizar a aquisição da imagem em microscópio óptico após a análise metalografica. A imagem de entrada será separada em sub-regiões contendo apenas um objeto de ferro fundido isolado, resultado do processo de segmentação da grafita. Cada divisão da imagem de entrada passará pelo processo de extração de atributos para, posteriormente, serem usadas no reconhecimento do tipo de ferro fundido. A análise destes objetos individuais permite o reconhecimento de cada amostra individualmente. Para reconhecer uma amostra com vários objetos, é reconhecido todos os objetos individualmente nesta imagem, sendo esta etapa chamada de "teste para a 
amostra toda" neste trabalho. O tipo de grafita que mais aparecer em uma amostra define a classificação adotada para toda amostra. Esta análise faz com que imperfeições na amostra não causem danos na classificação, onde objetos podem estar presente devido a inclusões no material e até mesmo por falhas na preparação na amostra.

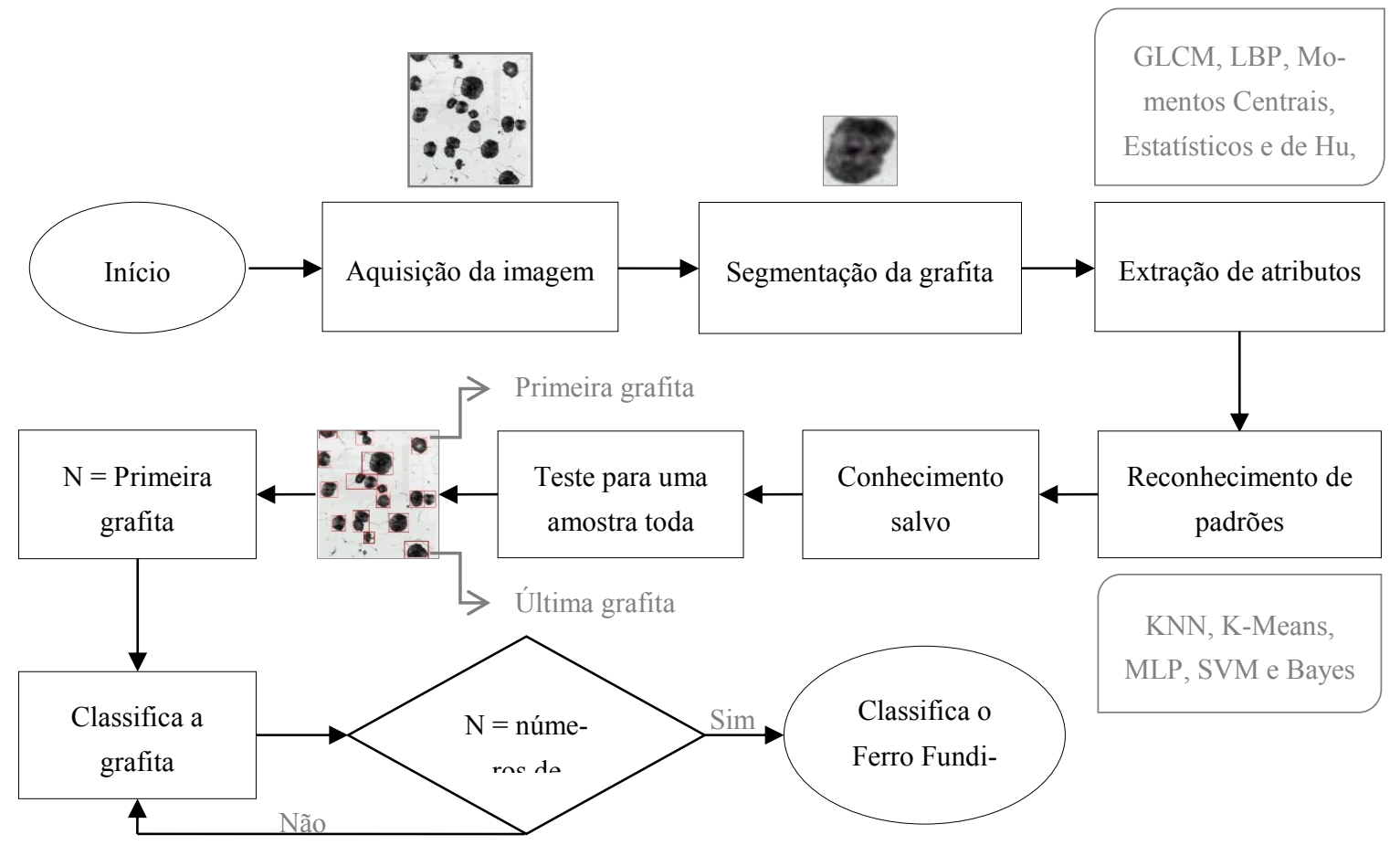

Figura 3: Fluxograma da metodologia adotada para as duas etapas.

A linguagem de programação utilizada foi a $\mathrm{C} / \mathrm{C}++$ através do ambiente integrado de desenvolvimento Visual Studio 2012. Este trabalho fez uso da biblioteca de código aberto OpenCV 3.0. O procedimento computacional foi realizado em um computador com processador Intel Core i5 de 2,4 GHz, 8 GB de RAM e sistema operacional Mac X El Capitan 10.11.2.

\subsection{Aquisição das imagens}

As imagens usadas neste trabalho foram adquiridas por meio de um microscópico ótico da marca Olympus do modelo GX51, com um parâmetro de aumento de 100 vezes. As dimensões dessas imagens foram de 640x480 pixels. Este procedimento foi realizado por especialistas no Laboratório de Materiais para Construção Mecânica do Instituto Federal do Ceará.

Inicialmente, foram obtidas amostras de ferros fundidos cinzento, maleável e nodular. Estas amostras foram cortadas em porções aproximadas de $13 \mathrm{~mm}$ de diâmetro e 13,6 mm de altura. Esse procedimento foi realizado em uma cortadora metalográfica da marca Teclago do modelo CM80, com discos abrasivos intensamente refrigerados, evitando deformações devido ao aquecimento, a relativas baixas rotações. Em seguida, houve o embutimento para facilitar o melhor manuseio nas etapas posteriores da metalografia. Foram realizados procedimentos com o objetivo de se retirar imperfeições mais profundas na superfície do material e deixá-la com uma superfície polida isenta de marcas. Durante esse processo foi necessário certificar-se, principalmente, das condições de iluminação e da preparação das amostras, pois, pode facilitar os procedimentos efetuados posteriormente.

\subsection{Teste para um objeto}

O processo de segmentação das grafitas foi realizado utilizando a segmentação por descontinuidade pela detecção de bordas do objeto, para isso se fez uso do operador gradiente Canny. Em seguida, foi aplicado os Contornos Conectados nas imagens com a utilização de uma matriz de busca de ordem 3.Para os contornos dos objetos ficarem melhores delimitados, foi empregado uma aproximação poligonal. 
Cada grafita de uma imagem foi segmentada e submetida a uma filtragem. Esse procedimento consiste em filtrar as grafitas de acordo com sua área, sendo assim, aquelas que apresentaram as maiores áreas foram utilizadas na etapa posterior, garantindo, desta forma, que as grafitas que possuíam áreas que pudessem ser confundidas com ruídos não comprometessem os resultados da análise. Após essa verificação, as grafitas consideradas como apropriadas, com relação a suas áreas, foram catalogadas como amostras dos ferros fundidos cinzento, maleável e nodular. De cada grafita resultante desse processo foi obtida uma imagem com o tamanho correspondente a grafita segmentada. Os objetos detectados com imperfeição no preparo da amostra foram descartados. Desde modo, foram eliminadas as imagens que não atendiam pré-requisitos de uma grafita.

Posteriormente ao processo de segmentação, foram utilizados cinco extratores de características em cada grafita segmentada. Foram usados 14, 48, 7, 10 e 7 números de atributos gerados pelos extratores GLCM, LBP, Momentos Centrais, Estatísticos e de Hu, respectivamente.

As bases de dados aplicadas neste trabalho foram obtidas usando 1700 grafitas de ferros fundidos dos três tipos trabalhados, sendo 331para o cinzento, 617 para o maleável e 752 para o nodular. Em seguida, esses dados dos extratores gerados foram classificados usando: KNN, SVM, MLP, Bayes e K-Means.

\subsection{Teste para uma amostra toda}

A etapa de aplicação do projeto foi realizada, primeiramente, a partir da obtenção das amostras de ferro fundido. Em seguida, é feita uma análise no microscópio ótico para averiguar se as imagens estão preparadas para serem submetidas ao sistema de classificação. Desde modo, verificam-se a iluminação e os resquícios da etapa de preparação do material na metalografia. Por fim, capturam-se as imagens das amostras utilizando uma câmera para que sejam submetidas ao sistema de classificação automatizada do tipo de ferro fundido.

A imagem que foi adquirida foi segmentada, sendo analisada considerando todos os objetos extraídos da mesma. Cada grafita é classificada entre ferro fundido nodular, maleável ou cinzento. $\mathrm{O}$ tipo de objeto com maior incidência na análise da imagem por completo é o reconhecimento adotado para toda a amostra.

\section{RESULTADOS E DISCUSSÕES}

Os resultados deste trabalho são apresentados particionados em duas etapas. Primeiramente, são mostrados os resultados obtidos pelos classificadores usados para o Reconhecimento de Padrões em que foram considerados cada objeto selecionado. Em seguida, são avaliados os resultados na qual a imagem completa foi analisada considerando todos os objetos extraídos da mesma, em que o tipo de objeto com maior incidência na análise seria o reconhecimento adotado para toda a amostra.

\subsection{Resultados do teste para um objeto}

A validação cruzada visa obter um conjunto de dados e subdividi-los aleatoriamente em dois subconjuntos diferentes: um utilizado para o treinamento e outro para o teste deste treinamento. Este processo irá realizar a validação do conjunto de teste a partir da análise do conjunto separado para o treinamento. Para atenuar a variabilidade, várias iterações de validação cruzada são realizadas.

Com relação a partição dos dados obtidos durante a etapa de extração, foram usadas duas abordagens para os testes, Hold Out e Leave One Out. No Hold Out, as amostras que foram destinadas para o conjunto de teste foram definidas aleatoriamente e o restantes das amostras que não foram escolhidas são determinadas para o treino. No Leave One Out, $80,0 \%$ das amostras de cada classe foram separadas para o conjunto de teste, e as demais reservadas para o treino.

Neste presente trabalho foram usadas 5 iterações para o Hold Out e Leave One Out, considerando $20,0 \%$ da amostra total de dados foram destinados ao conjunto de treino e 80,0\% para o conjunto de teste.

Para a etapa do Reconhecimento de Padrões, o classificador Bayes utiliza a distribuição normal. No classificador KNN a análise foi de 1, 3 e 5 vizinhos mais próximos. No classificador SVM foram considerados os kernels linear, RBF, polinomial e sigmóide. No K-Means foi usado a configuração com três grupos.

Para a rede MLP foram usadas topologias diferentes para cada dado de extração. Estas formatações foram determinadas avaliando com a configuração variando de 5 a 300 neurônios na camada oculta. Foram usados 25, 250, 90, 80, 35 neurônios nesta camada para os dados dos extratores dos Momentos de $\mathrm{Hu}$, Momentos Estatísticos e Momentos Centrais, GLCM e LBP, respectivamente. Com o intuito de validar os métodos de aprendizagem de máquinas, considerando as configurações utilizadas, foi feito uma análise dos valores das métricas sensibilidade, PPV, f-score e acurácia. As Tabelas 1 e 2 apresentam os resultados das métricas, obtidos para cada um dos classificadores utilizando as configurações especificadas. 
RODRIGUES. D.A.; SANTOS, G.P.; FERNANDES, M.C.; SANTOS, J.C.; FREITAS, F.N.C.; REBOUÇAS FILHO, P.P. revista Maté-

Tabela 1: Resultado das métricas obtidas por extração e classificação no Hold Out.

\begin{tabular}{|c|c|c|c|c|c|c|}
\hline \multicolumn{7}{|c|}{ HOLD OUT } \\
\hline Extrator & Classificador & Configuração & PPV (\%) & Sens (\%) & F-Score (\%) & Acc (\%) \\
\hline \multirow{11}{*}{ GLCM } & Bayes & Normal & $64,4 \pm 0,29$ & $64,4 \pm 0,29$ & $64,4 \pm 0,30$ & $68,9 \pm 0,30$ \\
\hline & \multirow{4}{*}{ SVM } & Linear & $95,7 \pm 0,00$ & $96,6 \pm 0,05$ & $96,1 \pm 0,01$ & $99,4 \pm 0,10$ \\
\hline & & RBF & $96,0 \pm 0,12$ & $96,7 \pm 0,12$ & $96,7 \pm 0,12$ & $97,8 \pm 0,44$ \\
\hline & & Polinomial & $96,3 \pm 0,50$ & $96,3 \pm 0,50$ & $96,3 \pm 0,50$ & $97,5 \pm 0,34$ \\
\hline & & Sigmóide & $44,2 \pm 0,23$ & $44,2 \pm 0,23$ & $44,2 \pm 0,23$ & $54,3 \pm 0,00$ \\
\hline & \multirow{2}{*}{ MLP } & Conf. 1 & $96,3 \pm 0,02$ & $96,0 \pm 0,01$ & $96,1 \pm 0,04$ & $97,5 \pm 0,27$ \\
\hline & & Conf. 2 & $96,6 \pm 0,03$ & $96,6 \pm 0,10$ & $96,6 \pm 0,08$ & $97,5 \pm 0,36$ \\
\hline & \multirow{3}{*}{$\mathrm{KNN}$} & $\mathrm{N}=1$ & $95,2 \pm 0,03$ & $94,0 \pm 0,01$ & $94,0 \pm 0,05$ & $95,2 \pm 0,01$ \\
\hline & & $\mathrm{N}=3$ & $95,7 \pm 0,01$ & $95,3 \pm 0,01$ & $95,5 \pm 0,01$ & $97,2 \pm 0,00$ \\
\hline & & $\mathrm{N}=5$ & $89,4 \pm 0,02$ & $97,9 \pm 0,01$ & $97,1 \pm 0,02$ & $99,6 \pm 0,03$ \\
\hline & \multicolumn{2}{|c|}{ K-Means } & $66,9 \pm 0,00$ & $63,2 \pm 0,00$ & $65,0 \pm 0,00$ & $75,2 \pm 0,00$ \\
\hline \multirow{11}{*}{ LBP } & Bayes & Normal & $84,4 \pm 0,91$ & $85,6 \pm 0,87$ & $85,0 \pm 0,92$ & $89,6 \pm 0,67$ \\
\hline & \multirow{4}{*}{ SVM } & Linear & $88,0 \pm 0,11$ & $87,1 \pm 0,09$ & $87,5 \pm 0,12$ & $91,7 \pm 0,79$ \\
\hline & & RBF & $93,2 \pm 0,61$ & $94,8 \pm 0,55$ & $94,0 \pm 0,10$ & $95,4 \pm 0,42$ \\
\hline & & Polinomial & $92,0 \pm 0,33$ & $95,3 \pm 0,18$ & $93,6 \pm 0,40$ & $94,5 \pm 0,23$ \\
\hline & & Sigmóide & $44,2 \pm 0,00$ & $40,2 \pm 0,01$ & $42,1 \pm 0,00$ & $54,3 \pm 0,00$ \\
\hline & \multirow{2}{*}{ MLP } & Conf. 1 & $86,7 \pm 0,70$ & $83,4 \pm 0,85$ & $85,0 \pm 0,60$ & $90,8 \pm 0,52$ \\
\hline & & Conf. 2 & $87,0 \pm 0,71$ & $86,9 \pm 0,72$ & $86,9 \pm 0,58$ & $91,0 \pm 0,52$ \\
\hline & \multirow{3}{*}{ KNN } & $\mathrm{N}=1$ & $90,0 \pm 0,05$ & $92,0 \pm 0,00$ & $90,7 \pm 0,01$ & $91,9 \pm 0,02$ \\
\hline & & $\mathrm{N}=3$ & $90,4 \pm 0,14$ & $91,3 \pm 0,10$ & $90,8 \pm 0,09$ & $93,3 \pm 0,10$ \\
\hline & & $\mathrm{N}=5$ & $90,7 \pm 0,02$ & $91,0 \pm 0,10$ & $90,8 \pm 0,04$ & $93,6 \pm 0,31$ \\
\hline & \multicolumn{2}{|c|}{ K-Means } & $44,3 \pm 0,00$ & $44,3 \pm 0,00$ & $44,3 \pm 0,00$ & $54,4 \pm 0,00$ \\
\hline \multirow{11}{*}{ M. Centrais } & Bayes & Normal & $31,7 \pm 0,40$ & $35,4 \pm 0,32$ & $33,4 \pm 0,20$ & $41,0 \pm 0,35$ \\
\hline & \multirow{4}{*}{ SVM } & Linear & $67,2 \pm 0,09$ & $67,2 \pm 0,09$ & $67,2 \pm 0,09$ & $70,0 \pm 0,10$ \\
\hline & & RBF & $57,5 \pm 0,29$ & $58,0 \pm 0,30$ & $57,7 \pm 0,20$ & $67,0 \pm 0,27$ \\
\hline & & Polinomial & $57,8 \pm 0,16$ & $57,8 \pm 0,19$ & $57,8 \pm 0,16$ & $67,3 \pm 0,15$ \\
\hline & & Sigmóide & $56,9 \pm 0,00$ & $58,0 \pm 0,00$ & $57,4 \pm 0,00$ & $66,4 \pm 0,00$ \\
\hline & \multirow{2}{*}{ MLP } & Conf. 1 & $59,8 \pm 0,36$ & $59,0 \pm 0,40$ & $59,4 \pm 0,32$ & $69,0 \pm 0,32$ \\
\hline & & Conf. 2 & $60,6 \pm 0,24$ & $61,3 \pm 0,32$ & $60,9 \pm 0,24$ & $69,0 \pm 0,21$ \\
\hline & \multirow{3}{*}{ KNN } & $\mathrm{N}=1$ & $45,9 \pm 0,09$ & $46,0 \pm 0,02$ & $45,0 \pm 0,05$ & $50,1 \pm 0,02$ \\
\hline & & $\mathrm{N}=3$ & $45,2 \pm 0,77$ & $48,0 \pm 0,50$ & $46,6 \pm 0,55$ & $55,3 \pm 0,77$ \\
\hline & & $\mathrm{N}=5$ & $51,9 \pm 1,05$ & $50,8 \pm 0,89$ & $51,3 \pm 0,90$ & $60,8 \pm 1,00$ \\
\hline & \multicolumn{2}{|c|}{ K-Means } & $56,9 \pm 0,00$ & $56,9 \pm 0,00$ & $56,9 \pm 0,00$ & $66,4 \pm 0,00$ \\
\hline \multirow{11}{*}{ M. HU } & Bayes & Normal & $25,0 \pm 0,00$ & $25,0 \pm 0,00$ & $25,0 \pm 0,00$ & $33,3 \pm 2,22$ \\
\hline & & Linear & $56,9 \pm 0,00$ & $56,8 \pm 0,00$ & $56,8 \pm 0,00$ & $66,4 \pm 0,00$ \\
\hline & SVM & RBF & $55,0 \pm 0,01$ & $55,1 \pm 0,00$ & $55,0 \pm 0,02$ & $66,4 \pm 0,00$ \\
\hline & SVM & Polinomial & $56,8 \pm 0,10$ & $56,8 \pm 0,02$ & $56,8 \pm 0,12$ & $66,4 \pm 0,11$ \\
\hline & & Sigmóide & $56,9 \pm 0,00$ & $59,0 \pm 0,00$ & $57,9 \pm 0,01$ & $66,4 \pm 0,00$ \\
\hline & & Conf. 1 & $57,6 \pm 0,50$ & $57,3 \pm 0,60$ & $57,4 \pm 0,45$ & $67,1 \pm 0,54$ \\
\hline & MLP & Conf. 2 & $57,3 \pm 1,23$ & $57,0 \pm 1,16$ & $57,1 \pm 1,20$ & $66,8 \pm 1,12$ \\
\hline & & $\mathrm{N}=1$ & $37,0 \pm 0,01$ & $40,0 \pm 0,02$ & $34,0 \pm 0,04$ & $49,0 \pm 0,07$ \\
\hline & KNN & $\mathrm{N}=3$ & $37,3 \pm 0,50$ & $37,3 \pm 0,54$ & $37,3 \pm 0,40$ & $47,8 \pm 0,57$ \\
\hline & & $\mathrm{N}=5$ & $41,0 \pm 0,72$ & $40,7 \pm 0,70$ & $40,8 \pm 0,67$ & $50,7 \pm 0,74$ \\
\hline & & & $35,4 \pm 0,00$ & $35,4 \pm 0,00$ & $35,4 \pm 0,00$ & $45,1 \pm 0,00$ \\
\hline & Bayes & Normal & $44,5 \pm 0,78$ & $44,5 \pm 0,80$ & $44,5 \pm 0,72$ & $54,6 \pm 0,78$ \\
\hline & & Linear & $61,0 \pm 3,19$ & $62,0 \pm 2,20$ & $61,5 \pm 3,00$ & $70,1 \pm 2,77$ \\
\hline & CVM & RBF & $64,0 \pm 3,25$ & $64,0 \pm 3,25$ & $64,0 \pm 3,25$ & $72,7 \pm 2,81$ \\
\hline & SVM & Polinomial & $62,9 \pm 5,04$ & $62,5 \pm 5,00$ & $62,7 \pm 5,01$ & $71,7 \pm 4,45$ \\
\hline & & Sigmóide & $57,7 \pm 0,61$ & $57,9 \pm 0,60$ & $57,8 \pm 0,59$ & $66,7 \pm 0,55$ \\
\hline M. Estatísticos & & Conf. 1 & $73,7 \pm 1,35$ & $73,8 \pm 1,40$ & $73,7 \pm 1,67$ & $86,7 \pm 1,63$ \\
\hline & MLP & Conf. 2 & $74,5 \pm 0,58$ & $74,9 \pm 0,57$ & $74,7 \pm 0,55$ & $81,4 \pm 0,46$ \\
\hline & & $\mathrm{N}=1$ & $55,4 \pm 0,02$ & $56,7 \pm 0,03$ & $53,9 \pm 0,03$ & $58,8 \pm 0,02$ \\
\hline & $\mathrm{KNN}$ & $\mathrm{N}=3$ & $55,5 \pm 0,47$ & $55,5 \pm 0,47$ & $55,5 \pm 0,47$ & $65,1 \pm 0,43$ \\
\hline & & $\mathrm{N}=5$ & $50,6 \pm 0,38$ & $51,0 \pm 0,40$ & $50,8 \pm 0,40$ & $60,6 \pm 0,36$ \\
\hline & & & $29,2 \pm 0,01$ & $29,5 \pm 0,01$ & $29,3 \pm 0,02$ & $37,0 \pm 0,02$ \\
\hline
\end{tabular}


RODRIGUES. D.A.; SANTOS, G.P.; FERNANDES, M.C.; SANTOS, J.C.; FREITAS, F.N.C.; REBOUÇAS FILHO, P.P. revista Maté-

Tabela 2: Resultado das métricas obtidas por extração e classificação no Leave One Out.

\begin{tabular}{|c|c|c|c|c|c|c|}
\hline \multicolumn{7}{|c|}{ LEAVE ONE OUT } \\
\hline Extrator & Classificador & Configuração & PPV (\%) & Sens $(\%)$ & F-Score $(\%)$ & $\operatorname{Acc}(\%)$ \\
\hline \multirow{11}{*}{ GLCM } & Bayes & Normal & $75,2 \pm 0,00$ & $75,0 \pm 0,00$ & $75,1 \pm 0,00$ & $81,9 \pm 0,00$ \\
\hline & \multirow{4}{*}{ SVM } & Linear & $94,4 \pm 0,19$ & $94,0 \pm 0,15$ & $94,2 \pm 0,18$ & $96,2 \pm 0,14$ \\
\hline & & RBF & $95,5 \pm 0,16$ & $95,8 \pm 0,14$ & $95,6 \pm 0,16$ & $97,0 \pm 0,11$ \\
\hline & & Polinomial & $95,0 \pm 0,63$ & $95,6 \pm 0,67$ & $95,3 \pm 0,66$ & $96,6 \pm 0,43$ \\
\hline & & Sigmóide & $44,2 \pm 0,00$ & $44,3 \pm 0,00$ & $44,2 \pm 0,00$ & $54,3 \pm 0,00$ \\
\hline & \multirow{2}{*}{ MLP } & Conf. 1 & $95,5 \pm 0,40$ & $95,5 \pm 0,40$ & $95,5 \pm 0,40$ & $97,0 \pm 0,27$ \\
\hline & & Conf. 2 & $95,0 \pm 0,47$ & $95,6 \pm 0,48$ & $95,3 \pm 0,50$ & $96,6 \pm 0,32$ \\
\hline & \multirow{3}{*}{ KNN } & $\mathrm{N}=1$ & $95,0 \pm 0,03$ & $95,0 \pm 0,02$ & $96,0 \pm 0,08$ & $95,0 \pm 0,01$ \\
\hline & & $\mathrm{N}=3$ & $94,1 \pm 0,00$ & $94,5 \pm 0,00$ & $94,3 \pm 0,00$ & $96,0 \pm 0,00$ \\
\hline & & $\mathrm{N}=5$ & $94,5 \pm 0,01$ & $94,3 \pm 0,02$ & $94,4 \pm 0,01$ & $96,3 \pm 0,00$ \\
\hline & \multicolumn{2}{|c|}{ K-Means } & $54,7 \pm 0,00$ & $54,7 \pm 0,00$ & $54,7 \pm 0,00$ & $64,4 \pm 0,00$ \\
\hline \multirow{11}{*}{ LBP } & Bayes & Normal & $84,7 \pm 0,00$ & $84,3 \pm 0,00$ & $84,5 \pm 0,00$ & $89,2 \pm 0,00$ \\
\hline & \multirow{4}{*}{ SVM } & Linear & $87,2 \pm 0,70$ & $87,5 \pm 0,65$ & $87,3 \pm 0,60$ & $91,0 \pm 0,51$ \\
\hline & & RBF & $91,0 \pm 0,34$ & $91,3 \pm 0,30$ & $91,1 \pm 0,40$ & $91,0 \pm 0,34$ \\
\hline & & Polinomial & $90,5 \pm 0,74$ & $90,5 \pm 0,74$ & $90,5 \pm 0,74$ & $93,5 \pm 0,53$ \\
\hline & & Sigmóide & $44,2 \pm 0,00$ & $44,5 \pm 0,01$ & $44,3 \pm 0,05$ & $54,3 \pm 0,00$ \\
\hline & \multirow{2}{*}{ MLP } & Conf. 1 & $88,3 \pm 1,12$ & $88,8 \pm 1,00$ & $88,5 \pm 1,02$ & $91,9 \pm 0,81$ \\
\hline & & Conf. 2 & $83,9 \pm 0,39$ & $85,0 \pm 0,25$ & $84,4 \pm 0,30$ & $88,7 \pm 0,29$ \\
\hline & \multirow{3}{*}{ KNN } & $\mathrm{N}=1$ & $91,3 \pm 0,04$ & $90,3 \pm 0,02$ & $90,0 \pm 0,01$ & $91,3 \pm 0,00$ \\
\hline & & $\mathrm{N}=3$ & $90,4 \pm 0,00$ & $91,0 \pm 0,10$ & $90,7 \pm 0,14$ & $90,4 \pm 0,00$ \\
\hline & & $\mathrm{N}=5$ & $90,4 \pm 0,00$ & $90,4 \pm 0,01$ & $90,4 \pm 0,50$ & $93,4 \pm 0,00$ \\
\hline & \multicolumn{2}{|c|}{ K-Means } & $44,3 \pm 0,01$ & $44,7 \pm 0,01$ & $44,5 \pm 0,01$ & $54,4 \pm 0,00$ \\
\hline \multirow{11}{*}{ M. Centrais } & Bayes & Normal & $33,8 \pm 0,01$ & $34,0 \pm 0,02$ & $33,9 \pm 0,00$ & $42,8 \pm 0,00$ \\
\hline & \multirow{4}{*}{ SVM } & Linear & $57,5 \pm 0,04$ & $58,0 \pm 0,07$ & $57,7 \pm 0,04$ & $67,0 \pm 0,04$ \\
\hline & & $\mathrm{RBF}$ & $57,3 \pm 0,09$ & $57,5 \pm 0,10$ & $57,4 \pm 0,04$ & $67,0 \pm 0,08$ \\
\hline & & Polinomial & $57,5 \pm 0,34$ & $57,9 \pm 0,39$ & $57,7 \pm 0,40$ & $66,9 \pm 0,31$ \\
\hline & & Sigmóide & $56,9 \pm 0,00$ & $57,0 \pm 0.00$ & $56,9 \pm 0,01$ & $66,4 \pm 0,00$ \\
\hline & \multirow{2}{*}{ MLP } & Conf. 1 & $58,2 \pm 1,00$ & $58,5 \pm 0,98$ & $58,3 \pm 0,93$ & $67,6 \pm 0,83$ \\
\hline & & Conf. 2 & $58,7 \pm 1,04$ & $59,0 \pm 1,09$ & $58,8 \pm 1,00$ & $67,7 \pm 0,94$ \\
\hline & \multirow{3}{*}{ KNN } & $\mathrm{N}=1$ & $45,0 \pm 0,02$ & $48,9 \pm 0,01$ & $47,0 \pm 0,02$ & $49,9 \pm 0,09$ \\
\hline & & $\mathrm{N}=3$ & $45,1 \pm 0,00$ & $45,1 \pm 0,00$ & $45,1 \pm 0,00$ & $55,2 \pm 0,00$ \\
\hline & & $\mathrm{N}=5$ & $45,8 \pm 0,01$ & $45,8 \pm 0,01$ & $45,8 \pm 0,01$ & $55,9 \pm 0,00$ \\
\hline & \multicolumn{2}{|c|}{ K-Means } & $56,8 \pm 0,06$ & $57,0 \pm 0,06$ & $56,9 \pm 0,10$ & $66,4 \pm 0,00$ \\
\hline \multirow{11}{*}{ M. HU } & Bayes & Normal & $36,9 \pm 0,03$ & $37,9 \pm 0,09$ & $37,4 \pm 0,10$ & $46,6 \pm 0,00$ \\
\hline & & Linear & $56,6 \pm 0,05$ & $56,9 \pm 0,05$ & $56,7 \pm 0,02$ & $66,2 \pm 0,00$ \\
\hline & SVM & RBF & $56,5 \pm 0,00$ & $57,0 \pm 0,00$ & $56,7 \pm 0,01$ & $66,1 \pm 0,00$ \\
\hline & SVIVI & Polinomial & $54,8 \pm 2,16$ & $55,0 \pm 2,11$ & $54,9 \pm 2,13$ & $64,5 \pm 2,01$ \\
\hline & & Sigmóide & $55,8 \pm 1,17$ & $56,0 \pm 1,10$ & $55,9 \pm 1,05$ & $65,6 \pm 1,08$ \\
\hline & MIP & Conf. 1 & $53,6 \pm 3,14$ & $54,0 \pm 3,50$ & $53,8 \pm 3,30$ & $63,4 \pm 2,97$ \\
\hline & VILF & Conf. 2 & $55,7 \pm 1,19$ & $56,0 \pm 1,15$ & $55,8 \pm 1,10$ & $65,3 \pm 1,10$ \\
\hline & & $\mathrm{N}=1$ & $39,9 \pm 0,01$ & $40,8 \pm 0,02$ & $37,8 \pm 0,04$ & $39,6 \pm 0,05$ \\
\hline & KNN & $\mathrm{N}=3$ & $36,8 \pm 0,00$ & $36,1 \pm 0,00$ & $36,4 \pm 0,00$ & $46,6 \pm 0,00$ \\
\hline & & $\mathrm{N}=5$ & $36,6 \pm 0,01$ & $36,7 \pm 0,00$ & $36,6 \pm 0,01$ & $46,4 \pm 0,00$ \\
\hline & & & $37,5 \pm 0,00$ & $37,5 \pm 0,00$ & $37,5 \pm 0,00$ & $47,4 \pm 0,00$ \\
\hline & Bayes & Normal & $46,6 \pm 0,00$ & $47,0 \pm 0,01$ & $46,8 \pm 0,03$ & $56,7 \pm 0,00$ \\
\hline & & Linear & $58,9 \pm 9,13$ & $59,0 \pm 9,10$ & $58,9 \pm 9,00$ & $68,0 \pm 3,39$ \\
\hline & & RBF & $65,2 \pm 0,02$ & $65,2 \pm 0,02$ & $65,2 \pm 0,02$ & $73,7 \pm 0,02$ \\
\hline & $S \vee M$ & Polinomial & $65,4 \pm 0,94$ & $65,4 \pm 0,94$ & $65,4 \pm 0,94$ & $73,9 \pm 0,79$ \\
\hline & & Sigmóide & $57,2 \pm 0,70$ & $57,6 \pm 0,75$ & $57,4 \pm 0,75$ & $66,7 \pm 0,63$ \\
\hline M. Estatísticos & MLE & Conf. 1 & $72,7 \pm 0,85$ & $72,3 \pm 0,87$ & $72,5 \pm 0,75$ & $80,0 \pm 0,68$ \\
\hline & VILP & Conf. 2 & $73,0 \pm 1,14$ & $73,4 \pm 1,10$ & $73,2 \pm 1,12$ & $80,3 \pm 0,92$ \\
\hline & & $\mathrm{N}=1$ & $55,9 \pm 0,00$ & $50,0 \pm 0,00$ & $52,4 \pm 0,02$ & $43,9 \pm 0,01$ \\
\hline & $\mathrm{KNN}$ & $\mathrm{N}=3$ & $49,1 \pm 0,00$ & $49,2 \pm 0,01$ & $49,1 \pm 0,00$ & $49,1 \pm 0,00$ \\
\hline & & $\mathrm{N}=5$ & $53,3 \pm 0,01$ & $53,7 \pm 0,01$ & $53,5 \pm 0,01$ & $63,2 \pm 0,00$ \\
\hline & & & $26,7 \pm 0,00$ & $26,4 \pm 0,00$ & $26,5 \pm 0,00$ & $35,3 \pm 0,00$ \\
\hline
\end{tabular}


A partir da análise das Tabelas 1 e 2, foi observado que os extratores GLCM e LBP obtiveram os melhores resultados. Estas taxas de acertos superiores se devem ao fato destes extratores gerarem dados para a classificação a partir da textura das imagens microscópicas. Já os resultados para os extratores Momentos de $\mathrm{Hu}$, Centrais e Estatísticos foram inferiores comparados aos extratores citados anteriormente. Isso é justificado pelo fato destes se basearem nas formas dos objetos, o que é um complicador devido à semelhança no formato entre objetos de ferros fundidos de diferente classificação.

É perceptível, pelas Tabelas 1 e 2, que o classificador SVM obteve os melhores resultados com o GLCM. Para os dados deste extrator, o classificador apresentou os melhores valores de acurácia no teste Hold Out, utilizando o kernel linear, e no teste Leave One Out, utilizando o kernel RBF. Ainda com base nestas observações, é possível verifica que o classificador KNN com uma análise dos cinco vizinhos mais próximos, apresenta valores altos de acurácia no teste Hold Out. Além da acurácia, essas mesmas combinações de extratores e classificadores apresentaram taxas expressivas das métricas de PPV, sensibilidade e fscore, desde modo, é validado a confiabilidade aos classificadores.

A Tabela 3 apresenta as matrizes de confusão percentuais das combinações do extrator com o classificador que obtiveram valores superiores a 90,0\% para todas as métricas. A partir da análise da Tabela 3 , nota-se que os classificadores avaliados obtiveram valores relevantes de corretas classificações em relação ao número total de amostras trabalhadas. Realizando uma análise geral das matrizes de confusão, notam-se resultados com excelentes percentuais de acertos quanto ao reconhecimento individual de cada classe.

Tabela 3: Matrizes de confusão percentuais do GLCM e LBP.

\begin{tabular}{|c|c|c|c|c|c|c|c|c|c|c|c|c|}
\hline \multicolumn{13}{|c|}{ MATRIZES DE CONFUSÃO } \\
\hline \multicolumn{13}{|c|}{ GLCM - Hold Out } \\
\hline & \multicolumn{3}{|c|}{ SVM Linear } & \multicolumn{3}{|c|}{ SVM RBF } & \multicolumn{3}{|c|}{ SVM Poly } & \multicolumn{3}{|c|}{ MLP 1} \\
\hline Classe & 1 & 2 & 3 & 1 & 2 & 3 & 1 & 2 & 3 & 1 & 2 & 3 \\
\hline 1 & 17,74 & 0,25 & 0,06 & 17,75 & 0,31 & 0 & 18,02 & 0,41 & 0,12 & 18,34 & 7,2 & 1,2 \\
\hline 2 & 1,23 & 35,31 & 0,5 & 1,15 & 35,17 & 0,57 & 1 & 35,22 & 0,88 & 0,73 & 35.18 & 1,07 \\
\hline \multirow[t]{2}{*}{3} & 0,43 & 0,81 & 43,67 & 0,5 & 0,9 & 43,66 & 0,38 & 0,73 & 43,23 & 0,32 & 0,66 & 43,07 \\
\hline & \multicolumn{3}{|c|}{ MLP 2} & \multicolumn{3}{|c|}{ KNN 1} & \multicolumn{3}{|c|}{ KNN 3} & \multicolumn{3}{|c|}{ KNN 5} \\
\hline Classe & 1 & 2 & 3 & 1 & 2 & 3 & 1 & 2 & 3 & 1 & 2 & 3 \\
\hline 1 & \begin{tabular}{|l|l}
17,99 \\
\end{tabular} & 0,29 & 0,12 & 18.02 & 0.30 & 0.30 & 17,49 & 0,37 & 0 & 17,41 & 0,29 & 0 \\
\hline 2 & 0,9 & 35,39 & 0,85 & 0.89 & 35.4 & 1.04 & 0,96 & 34,9 & 1,03 & 1,54 & 34,75 & 0,37 \\
\hline 3 & 0,51 & 0,69 & 43,26 & 0.23 & 1,20 & 42.62 & 0,96 & 1,1 & 43,2 & 0,44 & 1,32 & 43,86 \\
\hline \multicolumn{13}{|c|}{ GLCM - Leave One Out } \\
\hline & \multicolumn{3}{|c|}{ SVM Linear } & \multicolumn{3}{|c|}{ SVM RBF } & \multicolumn{3}{|c|}{ SVM Poly } & \multicolumn{3}{|c|}{ MLP 1} \\
\hline Classe & 1 & 2 & 3 & 1 & 2 & 3 & 1 & 2 & 3 & 1 & 2 & 3 \\
\hline 1 & 17,94 & 0,15 & 0,12 & 18 & 0,18 & 0,12 & 18,07 & 0,15 & 0,15 & 18,1 & 0,69 & 0,03 \\
\hline 2 & 0,97 & 32,59 & 0,22 & 0,91 & 33,55 & 0,15 & 0,88 & 33,03 & 0,16 & 0,87 & 33,61 & 0,44 \\
\hline \multirow[t]{2}{*}{, } & 0,48 & 3,63 & 43,89 & 0,48 & 2,65 & 43,97 & 0,44 & 3,19 & 43,92 & 0,43 & 2,07 & 43,76 \\
\hline & \multicolumn{3}{|c|}{ MLP 2} & \multicolumn{3}{|c|}{ KNN 1} & \multicolumn{3}{|c|}{ KNN 3} & \multicolumn{3}{|c|}{ KNN 5} \\
\hline Classe & 1 & 2 & 3 & 1 & 2 & 3 & 1 & 2 & 3 & 1 & 2 & 3 \\
\hline 1 & 18,22 & 0,97 & 0,06 & 18.02 & 0.40 & 0.03 & 17,05 & 0,37 & 0 & 17,34 & 0,44 & 0 \\
\hline 2 & 0,82 & 33,14 & 0,51 & 2,00 & 35,09 & 0,46 & 1,54 & 33,14 & 0,29 & 1,32 & 33,14 & 0,22 \\
\hline 3 & 0,35 & 2,26 & 43,66 & 0.90 & 3.01 & 40,09 & 0,81 & 2,87 & 43,97 & 0,73 & 2,79 & 44,01 \\
\hline \multicolumn{13}{|c|}{ LBP - Hold Out } \\
\hline & \multicolumn{3}{|c|}{ SVM Poly } & \multicolumn{3}{|c|}{ SVM RBF } & \multicolumn{3}{|c|}{ KNN 3} & & KNN 5 & \\
\hline Classe & 1 & 2 & 3 & 1 & 2 & 3 & 1 & 2 & 3 & 1 & 2 & 3 \\
\hline 1 & 17,8 & 1,43 & 0,04 & 17,12 & 1,37 & 0,03 & 16,72 & 1,19 & 0,12 & 16,25 & 1,16 & 0 \\
\hline 2 & 1,47 & 32,76 & 1,48 & 2,19 & 33,08 & 1,63 & 2,32 & 32,15 & 1,72 & 2,82 & 31,45 & 1,54 \\
\hline 3 & 0,13 & 2,19 & 42,7 & 0,09 & 1,93 & 42,57 & 0,35 & 3,03 & 42,4 & 0,32 & 3,76 & 42,69 \\
\hline & & & & & LBP - I & eave $\mathrm{O}$ & Out & & & & & \\
\hline & & $\mathrm{VM} \mathrm{Pol}$ & & & VM RB & & & KNN 3 & & & KNN 5 & \\
\hline Classe & 1 & 2 & 3 & 1 & 2 & 3 & 1 & 2 & 3 & 1 & 2 & 3 \\
\hline 1 & 18,53 & 2,48 & 0,07 & 17,56 & 2,09 & 0,07 & 17,27 & 1,4 & 0 & 18.00 & 2.00 & 1.98 \\
\hline 2 & 0,57 & 28,95 & 0,69 & 1,62 & 29,96 & 1,15 & 1,76 & 30,57 & 1,69 & 0.8 & 15.8 & 1,80 \\
\hline 3 & 0,29 & 4,94 & 43,47 & 0,22 & 4,32 & 43,01 & 0,37 & 4,41 & 42,54 & 9.91 & 5.90 & 43.90 \\
\hline
\end{tabular}

Com base nas informações constantes nas matrizes de confusão para cada um dos classificadores, combinados a cada extrator, foi possível aferir taxas de acerto, precisão e confiabilidade dos métodos. Como 
pode ser analisado na matriz de confusão na Tabela 3, um dos classificadores de destaque é o SVM com o kernel RBF para o teste Leave One Out, na qual, os valores da diagonal principal indicam excelentes resultados, com a classe 1 apresentando percentual de acerto de $18 \%$, enquanto as classes 2 e 3 apresentam 33,55\% e $43,97 \%$, respectivamente, totalizando o valor de $95,52 \%$. Este percentual total combinado aos baixos percentuais de erros que cada classe apresenta confere a este classificador um alto desempenho.

\subsection{Resultados do teste para uma amostra toda}

Os resultados são obtidos a partir das amostras de ferro fundido cinzento, maleável e nodular, em que todas são submetidas a um reconhecimento de grafita por amostra possibilitando uma verificação do reconhecimento automático do tipo de ferro fundido. Para todas as amostras foram utilizados os classificadores que alcançaram valores superiores a 90,0\% nas métricas avaliadas durante o teste para um objeto.

São mostrados na Figura 4 os resultados obtidos com a aplicação do reconhecimento de grafita por amostra, sendo que a classe 1, classe 2 e classe 3 correspondem a ferro fundido cinzento, maleável e nodular, respectivamente. Nota-se que os resultados gerais dos gráficos apresentam valores superiores a 90,0\%. Constata-se também que os resultados obtidos para as amostras de ferro fundido cinzento e nodular apresentam uma pequena superioridade com relação ao reconhecimento do ferro fundido maleável. Isto porque as grafitas das amostras são visualmente mais bem definidas.

Analisando os resultados descritos na Figura 4, percebe-se a eficácia do classificador SVM com o kernel para RBF. Este se destacou com o reconhecimento de cerca de 100,0\% dos ferros fundidos do tipo cinzento, maleável e nodular no extrator GLCM. Já para o LBP, o mesmo teve um acerto de 100,0\% para o cinzento e nodular, possuindo apenas uma falha na amostra do ferro fundido maleável.

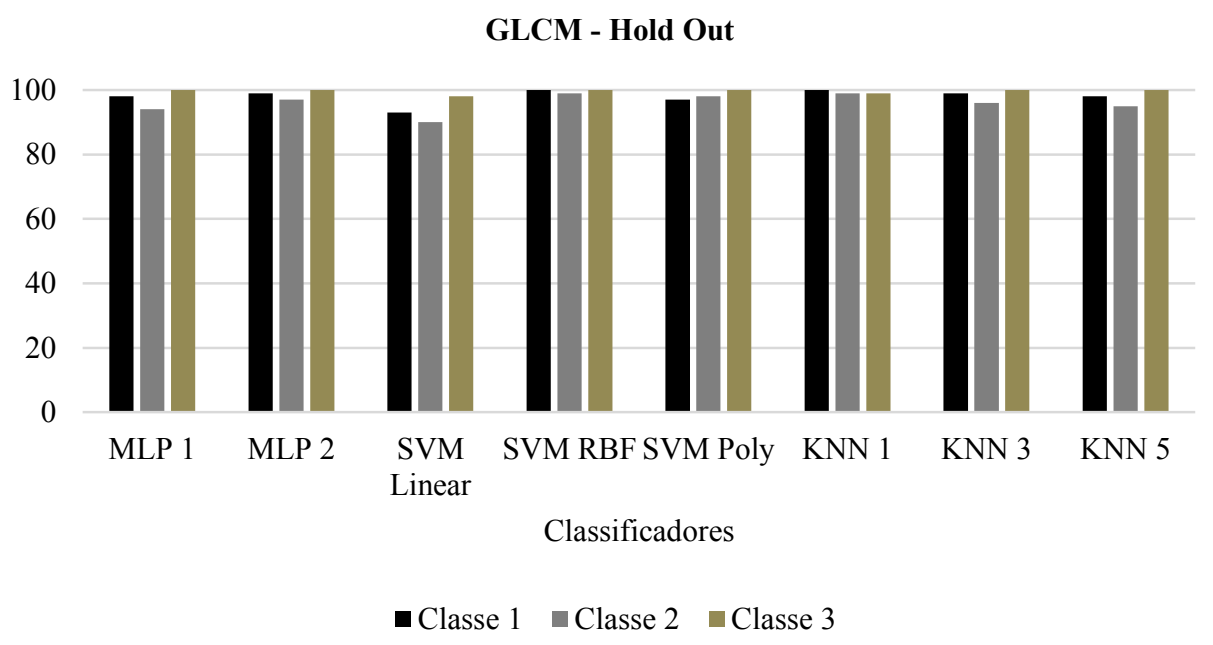

a)

GLCM - Leave One Out

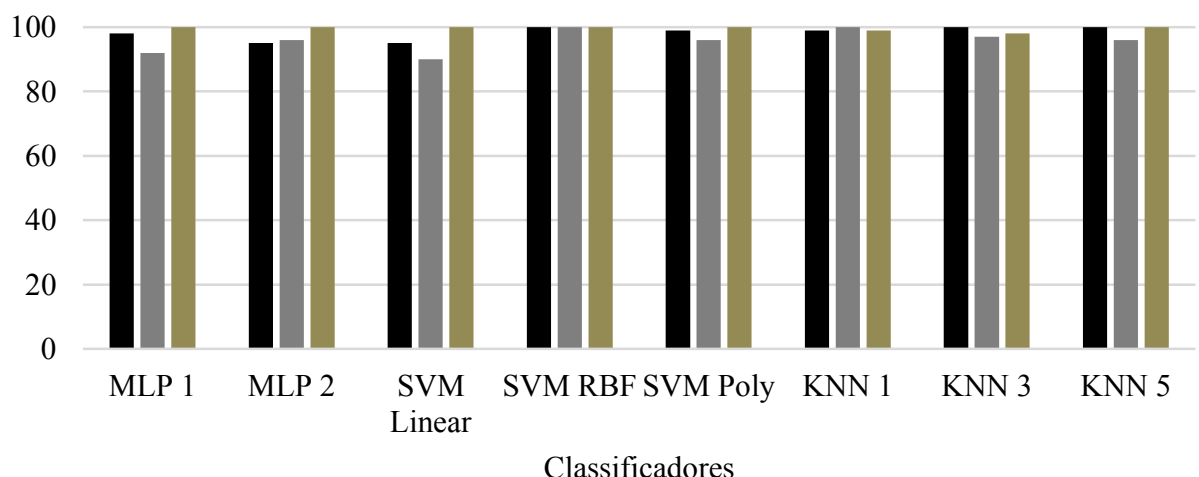

- Classe 1 - Classe 2 Classe 3

b) 


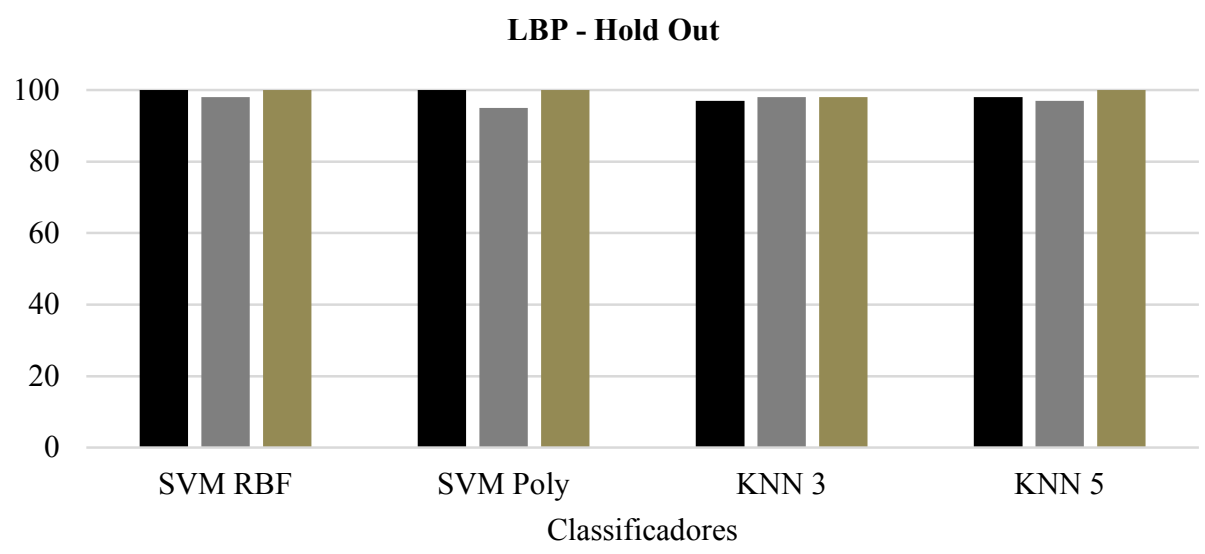

- Classe 1 - Classe 2 - Classe 3

c)

\section{LBP - Leave One Out}

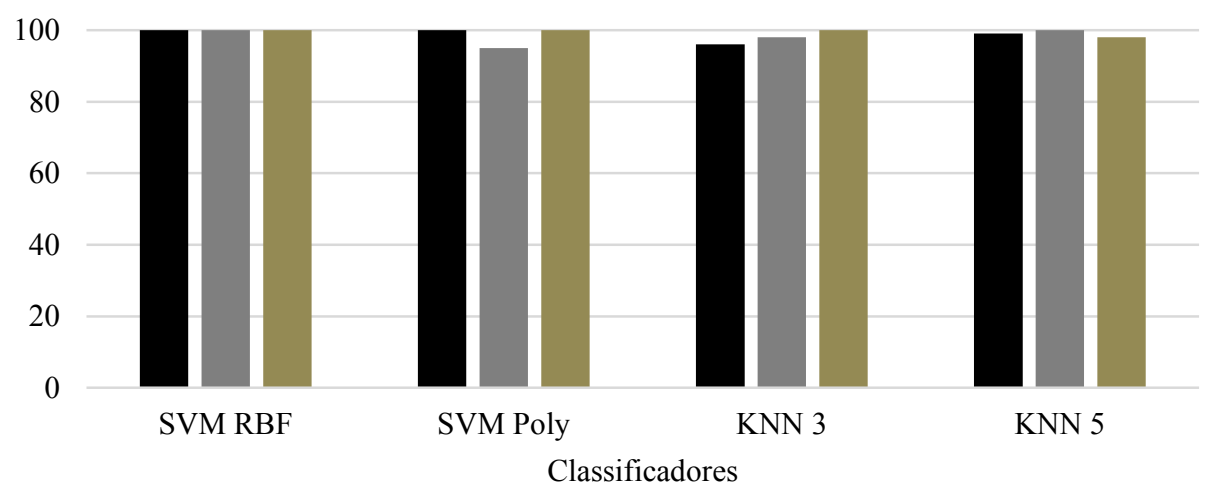

- Classe $1 \quad$ - Classe 2 Classe 3

d)

Figura 4: Resultados gerais do teste para uma amostra toda utilizando o reconhecimento de padrão para o: a) GLCM com o Hold Out; b) GLCM com o Leave One Out; c) LBP com o Hold Out; d) LBP com o Leave One Out.

Os resultados gerados pelo sistema computacional de classificação foram comparados aos resultados obtidos pelos métodos tradicionais de análise realizados por um especialista. Para essa comparação, foi empregado o classificador de maior destaque entre os resultados apresentados na Figura 4. Desde modo, foi utilizado o classificador SVM com o kernel RBF com o extrator GLCM. Para a validação dos resultados gerados utilizando a análise do especialista, algumas amostras de ferros fundidos que foram submetidas no teste para uma amostra toda foram analisadas visualmente pelo profissional.

Os resultados observados com o classificador e o extrator utilizado para a classificação do ferro fundido estão de acordo com a classificação do especialista. No entanto, houve uma considerável variação no tempo para a realização das classificações dessas amostras. São mostrados na Tabela 4 os tempos médios de classificação para cada tipo de ferro fundido realizado pelo especialista, de modo tradicional, e o com a aplicação do procedimento automático.

O procedimento automático apresenta a vantagem da redução considerável do tempo de classificação em relação a análise visual. Visto que, estes possuem tempo de classificação médio bastante inferior que aos procedimentos convencionais de classificação. O aumento considerável do tempo de análise do especialista é motivado pela dificuldade encontrada em algumas amostras na caracterização da forma da grafita, o que requer maior tempo de análise visual. 
Tabela 4: Tempo médio em segundos de classificação do ferro fundido para a classificação automática e para a análise visual. Sendo que a classe 1, 2 e 3 corresponde ao ferro fundido cinzento, maleável e nodular, respectivamente.

\begin{tabular}{c|c|c}
\hline \multicolumn{3}{c}{ AVALIAÇÃo COMPARATIVA DO TEMPO DE CLASSIFICAÇÃo } \\
\hline Classe & Procedimento automático & Análise Visual \\
\hline 1 & 6,08 & 46,8 \\
\hline 2 & 2,41 & 36,0 \\
\hline 3 & 1,66 & 34,4 \\
\hline
\end{tabular}

Realizando uma análise comparativa entre os resultados apresentados neste trabalho e os já apresentados em trabalhos anteriores, certifica-se novamente a eficácia da classificação dos tipos de ferros fundidos com o uso da Inteligência Computacional Aplicada. Dado que, no trabalho de GOMES e PACIONIRK [18], o percentual de acerto para os ferros fundidos foi cerca de a 90\%. Já os resultados de classificações corretas apresentados no trabalho de PATTAN et al. [19], oscilaram em uma faixa entre $73 \%$ e $87 \%$.

\section{CONCLUSÕES}

O presente trabalho apresenta uma inovadora metodologia com o uso da Inteligência Computacional Aplicada para realizar a classificação automática entre os tipos de ferros fundidos. Tradicionalmente, essa classificação é realizada a partir de uma rígida e atenciosa análise visual da metalografia do ferro fundido, realizada por um profissional, requerendo um tempo considerável para se realizar essas classificações. O sistema automático implementado contribui com êxito na classificação dos tipos ferro fundido, reduzindo consideravelmente o tempo necessário para se realizar essa categorização, além de restringir as falhas na classificação decorrentes das limitações humanas.

Em relação as tecnologias utilizadas para classificação dos objetos, o classificador SVM obteve os melhores resultados, obtendo 99,4 $\pm 0,10$ de acurácia no teste Hold Out com o ajusto do kernel para o linear, e $97,0 \pm 0,11$ no teste Leave One Out utilizando o kernel RBF. Já para análise da amostra como um todo, o classificador SVM com kernel RBF obteve os melhores resultados no reconhecimento do tipo de ferro fundido, reconhecendo com um percentual de quase $100,0 \%$ os ferros fundidos maleável, cinzento e nodular. A partir destes resultados, pode ser observado que houve um acréscimo no percentual de acerto de ferros fundidos corretamente classificados se comparados aos trabalhos que utilizam uma abordagem com redes neurais $\mathrm{e}$ uma classificação supervisionada utilizando apenas os descritores de forma.

A partir da análise dos resultados apresentados, pode-se concluir que a abordagem é promissora, podendo incorporar softwares comerciais para auxílio à engenheiros, especialistas, estudantes e outros da área da Ciências dos Materiais. Para trabalhos futuros, pretende-se expandir este reconhecimento para outros tipos de ferros fundidos, como o ferro fundido branco. Além do uso de outros extratores e classificadores.

\section{AGRADECIMENTOS}

Os autores agradecem ao apoio financeiro do IFCE via editais de fomento PROAPP-IFCE de 2014, PAPEX/IFCE de 2015 e PROFEP/IFCE-Maracanaú de 2016.

\section{BIBLIOGRAFIA}

[1] PEVEC, M., ODER, G., POTRC, I., et al., "Elevated temperature low cycle fatigue of grey cast iron used for automotive brake discs", Engineering Failure Analysis, v. 42, pp. 221-230, July 2014.

[2] JOTSHI, C.K., et al., "Heat transfer characteristics of a high temperature sensible heat storage water heater using cast iron as a storage material", In: Energy Conversion Engineering Conference, 1996. IECEC 96. Proceedings of the 31st Intersociety, pp. 2099-2103, Washington, DC, USA, Aug. 1996.

[3] CRISAN, A., MUNTEANU, S.I., CIOBANU, I., et al., "Optimization of the chemical composition of cast iron used for casting ball bearing grinding discs", Tsinghua Science \& Technology, v. 13, n. 2, pp. 164-169, Apr. 2008.

[4] CHIAVERINI, V., Aços e Ferros Fundidos, 7 ed., São Paulo, Associação Brasileira de Metais - ABM, 1996.

[5] CALLISTER, W.D.Jr., Ciência e Engenharia de Materiais: Uma introdução, 8 ed., Rio de Janeiro, LTC, 2012. 
RODRIGUES. D.A.; SANTOS, G.P.; FERNANDES, M.C.; SANTOS, J.C.; FREITAS, F.N.C.; REBOUÇAS FILHO, P.P. revista Maté-

[6] VÉRTESY, G., UCHIMOTO, T., TAKAGI, T., et al., "Flake Graphite Cast Iron Investigated by a Magnetic Method", IEEE Transactions on Magnetics, v. 50, n. 4, pp. 1-4, Apr. 2014.

[7] PAPA, J.P., ALBUQUERQUE, V.H.C., FALCÃO, A.X., et al., "Fast automatic microstructural segmentation of ferrous alloy samples using optimum-path Forest", In: Computational Modeling of Objects Represented in Images, pp. 210-220, Buffalo, New York, USA, May 2010.

[8] PAPA, J.P., NAKAMURA, R.Y.M., ALBUQUERQUE, V.H.C., et al., "Computer techniques towards the automatic characterization of graphite particles in metallographic images of industrial materials", Expert Systems with Applications, v. 40, n. 2, pp. 590-597, Jul. 2013.

[9] ALBUQUERQUE, V.H.C., BARBOSA, C.V., SILVA, C.C., et al., "Ultrasonic sensor signals and optimum path forest classifier for the microstructural characterization of thermally-aged Inconel 625 alloy", Sensors, v. 15, n. 6, pp. 12474-12497, May 2015.

[10] REBOUÇAS FILHO, P.P., MOREIRA, F.D.L., XAVIER, F.G.L., et al., "New analysis method application in metallographic images through the construction of mosaics via speeded up robust features and scale invariant feature transform", Materials, v. 8, n. 7, pp. 3864-3882, Jun. 2015.

[11] ALBUQUERQUE, V.H.C., CORTEZ, P.C., ALEXANDRIA, A.R., et al., "A new solution for automatic microstructures analysis from images based on a backpropagation artificial neural network", Nondestructive Testing and Evaluation, v. 23, n. 4, pp. 273-283, Jun. 2008.

[12] ALBUQUERQUE, V.H.C., TAVARES, J.M.R.S., CORTEZ, P.C., et al., "Quantification of the microstructures of hypoeutectic white cast iron using mathematical morphology and an artificial neural network", International Journal of Microstructure and Materials Properties, v. 5, n. 1, pp. 52-64, Jun. 2010.

[13] ALBUQUERQUE, V.H.C., NAKAMURA, R. Y.M., PAPA, J.P., et al., "Automatic segmentation of the secondary austenite-phase island precipitates in a super duplex stainless steel weld metal", In: III ECCOMAS Thematic Conference on Computational Vision and Medical Image Processing: Vip IMAGE 2011, pp. 161166, Olhão, Algarve, Portugal, Oct. 2011.

[14] ALBUQUERQUE, V.H.C., ALEXANDRIA, A.R., CORTEZ, P.C., et al., "Evaluation of multilayer perceptron and self-organizing map neural network topologies applied on microstructure segmentation from metallographic images", NDT \& E International, v. 42, n. 7, pp. 644-651, May 2009.

[15] REBOUÇAS, E.S., BRAGA, A.M., MARQUES, R.C.P., et al., "A new approach to calculate the nodule density of ductile cast iron graphite using a level set”, Measurement, v. 89, n. 2, pp. 316-321, Apr. 2016.

[16] PEIXOTO, F.M.A., REBOUÇAS, E.S., XAVIER, F.G.L., et al., "Desenvolvimento de um Software para cálculo da densidade de nódulos de grafita em ferro fundido nodular através de Processamento Digital de Imagens", Matéria (UFRJ), v. 20, n. 1, pp. 262-272, Mar. 2015.

[17] ALBUQUERQUE, V.H.C., CORTEZ, P.C., ALEXANDRIA, A.R., et al., "Sistema de segmentação de imagens para quantificação de microestruturas em metais utilizando redes neurais artificiais", Matéria (UFRJ), v. 12, n. 2, pp. 394-407, Mar. 2007.

[18] GOMES, O., PACIORNIK, S. "Automatic Classification of Graphite in Cast Iron “, Microscopy and Microanalysis, v.11, n. 4, pp. 363-371, Feb. 2005.

[19] PATTAN, P.C., MYTRI, V., HIREMATH, P. "Classification of cast iron based on graphite grain morphology using neural network approach", In: Second International Conference on Digital Image Processing, pp. 75462S-1-75462S-6, Singapore, Singapore, Feb. 2010.

[20] ALBERTO, S.A., MARK, S.N. Feature extraction and image processing, 3 ed., Massachusetts, USA, Newnes, 2002.

[21] GONZALEZ, R.C., WOODS, R.E., Digital Image Processing, 3 ed., São Paulo, Pearson, 2008.

[22] CANNY, J. “A computational approach to edge detection", IEEE Transactions on Pattern Analysis and Machine Intelligence, v. 8, n. 6, pp. 679-698, Nov. 1986.

[23] GOMES, S.L., REBOUÇAS, P.P., REBOUÇAS, E.S., et al., "Computer Vision System to Aid Drivers of Vehicles through Vertical Signaling Traffic", International Journal of Computer Applications, v. 115, n. 5 , pp. 1-7, Apr. 2015.

[24] CZACHORSKI, T., KOZIELSKI, S., STANCZYK, U. Man-Machine Interactions 2, 1 ed., SpringerVerlag, Heidelberg, Germany, 2011.

[25] HU, M.-K. "Visual pattern recognition by moment invariants", IRE Transactions on Information Theory, v. 8, n. 2, pp. 179-187, Feb. 1962. 
RODRIGUES. D.A.; SANTOS, G.P.; FERNANDES, M.C.; SANTOS, J.C.; FREITAS, F.N.C.; REBOUÇAS FILHO, P.P. revista Maté-

[26] FLUSSER, J., SUK, T., BOLDYS, J., et al., "Projection Operators and Moment Invariants to Image Blurring", IEEE Transactions on Pattern Analysis and Machine Intelligence, v. 37, n. 4, pp. 786-802, Apr. 2015.

[27] LICCIARDI, G., VILLA, A., MURA, M.D., et al., "Retrieval of the Height of Buildings From WorldView-2 Multi-Angular Imagery Using Attribute Filters and Geometric Invariant Moments", IEEE Journal of Selected Topics in Applied Earth Observations and Remote Sensing, v. 5, n. 1, pp. 71-79, Feb. 2012.

[28] YANG, J., XIONG, N., VASILAKOS, A.V., et al., "A Fingerprint Recognition Scheme Based on Assembling Invariant Moments for Cloud Computing Communications", IEEE Systems Journal, v. 5, n. 4, pp. 574-583, Dec. 2011.

[29] HARALICK, R. "Statistical and structural approaches to texture", Proceedings of the IEEE, v. 67, n. 5, pp. 786-804, May 1979.

[30] HARALICK, R., SHANMUGAM, K., DINSTEIN, I. "Textural Features for Image Classification", IEEE Transactions on Systems, Man, and Cybernetics, v. 3, n. 6, pp. 610-621, Nov. 1973.

[31] RAMALHO, G.L.B., REBOUÇAS FILHO, P.P., MEDEIROS, F.N.S., et al., "Lung disease detection using feature extraction and extreme learning machine", Revista Brasileira de Engenharia Biomedica, v. 30, n. 3, pp. 207-214, Sept. 2014.

[32] CAVALCANTI NETO, E., et al., "Supervised Enhancement Filter Applied to Fissure Detection", In: VI Latin American Congress on Biomedical Engineering CLAIB 2014, pp. 337-340, Paraná, Entre Rios, Argentina, Oct. 2014.

[33] OJALA, T., PIETIKAINEN, M., MAENPAA, T. "Multiresolution gray-scale and rotation invariant texture classification with local binary patterns", IEEE Transactions on Pattern Analysis and Machine Intelligence, v. 24, n. 7, pp. 971-987, Jul. 2002.

[34] FACELI, K., et al., Inteligência artificial: Uma abordagem de aprendizado de máquina, 1 ed., São Paulo, LTC, 2011.

[35] BISHOP, C., Pattern Recognition and Machine Learning, 1 ed., New York, USA, Springer, 2006.

[36] MITCHELL, T.M., Machine Learning, 1 ed., Boston, Massachusetts, USA, McGraw-Hill, 1997.

[37] VAPNIK, V.N., "An overview of statistical learning theory”, IEEE Transactions on Neural Networks, v. 10, n. 5, pp. 988-999, Sept. 1999.

[38] CRAMMER, K., SINGER, Y., "On the Algorithmic Implementation of Multiclass Kernel-based Vector Machines”, Journal of Machine Learning Research, v. 2, n. 1, pp. 265-292, Dec. 2001.

[39] DUAN, K.-B., KEERTHI, S.S., "Which Is the Best Multiclass SVM Method? An Empirical Study”, In: International Workshop on Multiple Classifier Systems, pp. 278-285, Seaside, California, USA, June 2005.

[40] RUCK, D.W., ROGERS, S.K., KABRISKY, M., et al., "The Multilayer Perceptron as an Approximation to a Bayes Optimal Discriminant Function”, IEEE Transactions on Neural Networks, v. 1, n. 4, pp. 296-298, Dec. 1990.

[41] HAYKIN, S.O., Neural Networks and Learning Machines, 3 ed., New Jersey, Pearson, 2008.

[42] MINSKY, M., PAPERT, S., Perceptrons: An Introduction to Computational Geometry, 4 ed., Massachusetts, USA, MIT Press, 1988.

[43] MEDEIROS, C.M.S., Uma Contribuição ao problema de seleção de modelo neurais usando o princípio de máxima correlação dos erros, Tese D.Sc., Universidade Federal do Ceará, Fortaleza, 2008. 